\title{
On Presupposition Projection with Trivalent Connectives*
}

\author{
Yoad Winter \\ Utrecht University
}

\begin{abstract}
A basic puzzle about presuppositions concerns their projection from propositional constructions. This problem has regained much attention in the last decade since many of its prominent accounts, including variants of the trivalent Strong Kleene connectives, suffer from the so-called proviso problem. This paper argues that basic insights of the Strong Kleene system can be used without invoking the proviso problem. It is shown that the notion of determinant value that underlies the definition of the Strong Kleene connectives leads to a natural generalization of the filtering conditions proposed in Karttunen's article "Presuppositions of compound sentences" (LI, 1973). Incorporating this generalized condition into an incremental projection algorithm avoids the proviso problem as well as the derivation of conditional presuppositions. It is argued that the same effects that were previously modelled using conditional presuppositions may be viewed as effects of presupposition suspension and contextual inference on presupposition projection.
\end{abstract}

Keywords: presupposition, trivalence, connectives, proviso, projection, filtering, Kleene

\section{Introduction}

It is now almost fifty years since Karttunen (1973) observed that different propositional operators show different regularities with presupposition projection. Karttunen focused on examples like the following:

(1) a. Jack has children (\#no children) and all of Jack's children are bald.

b. Jack has no children (\#children) or (else) all of Jack's children are bald.

In these sentences, the second conjunct ("all of Jack's children are bald") presupposes that Jack has children. In conjunctions like (1a), this presupposition disappears

* A technical version of central ideas in this paper appeared in (Winter 2019). The analysis of conditional presuppositions is new with the present version. For their comments, I am grateful to three MOL reviewers, as well as to Lisa Bylinina, Donka Farkas, Danny Fox, Itamar Francez, Philippe de Groote, James Hampton, Lauri Karttunen, Ed Keenan, Heidi Klockmann, Matthew Mandelkern, Rick Nouwen, Jacopo Romoli, Philippe Schlenker, members of the presupposition reading group in Utrecht, and the SALT 29 audience. Work on this paper was partially funded by the European Research Council (ERC) under the European Union's Horizon 2020 research and innovation programme (grant agreement No 742204).

(C)2019 Winter 
when the first conjunct asserts it. By contrast, in disjunctions like (1b), the same presupposition disappears when the first conjunct negates it. Contrasts similar to (1a-b) have led semanticists to hypothesize that the way in which presuppositions are "projected" or "filtered" should be predictable from the core meanings of the propositional connectives. At the same time, Karttunen's findings exposed a general problem for standard treatments of propositional operators in trivalent semantics. Whether presuppositions are filtered or projected in propositional constructions depends on semantic and pragmatic relations between the operands, and not only on their truth-values in specific models. This point challenges purely truth-conditional accounts of presupposition in trivalent semantics (van Fraassen 1971). A similar problem was pointed out in later years (Geurts 1996) for dynamic frameworks involving context changing operators (Stalnaker 1973; Karttunen 1974; Heim 1983), and was branded as the proviso problem. Recent works continue to address this problem (van Rooij 2007; Singh 2007; Schlenker 2011; Lassiter 2012; Mayr \& Romoli 2016; Mandelkern 2016; Mandelkern \& Rothschild 2018; Francez 2019).

Taking a step back from dynamic accounts, and in the face of the proviso problem, this paper reconsiders the trivalent treatment of presuppositions as a truthconditional phenomenon. As it turns out, this treatment allows us to formally regain a unified outlook on different propositional operators using one general filtering condition. This condition takes into account Karttunen's misgivings about truth-functional accounts of presupposition projection, while synthesizing them with more recent ideas about incremental processing (Schlenker 2008, 2009; Fox 2008). This approach readopts a key insight of Strong Kleene operators: values that determine the result of a bivalent operator also determine the result of its trivalent version. It is shown that this approach can completely avoid the proviso problem when implemented as a global condition on filtering following Karttunen's original observations. A question that this proposal leaves open concerns the status of socalled "conditional presuppositions" (Beaver 2001). These are cases where the presupposition of a propositional construction is weaker than the presupposition of the triggering operand, but the latter does not seem to be completely filtered. I will argue that the proposed filtering condition allows us to accommodate such effects without running into the proviso problem. A key observation of Karttunen's work is that conditional presuppositions can be explained as resulting from the sensitivity of filtering to contextual inferences. We treat these contextual inferences by quantifying over intended models as part of the general filtering condition. This, together with the well-known observation (Gazdar 1979; Soames 1982; Abbott 2006) about the possibility of presuppositions to be "suspended" under pragmatic pressure, is claimed to give a non-presuppositional account of conditional inferences. Since proviso problems result from techniques that modify presuppositions into conditional presuppositions, it is concluded that such techniques may be empirically unnecessary 
and that full filtering/projection of presuppositions may be sufficient. At the same time, it is suggested that a major open challenge for trivalent semantics lies in the need to selectively filter presuppositions of certain triggers in an expression, while fully projecting presuppositions of other triggers in the same expression.

Section 2 reviews trivalent approaches to presupposition projection, with the Strong Kleene truth tables as a dominant tool, leading to the Schlenker/Fox formulation of incremental truth-valuation. This proposal, despite its attractiveness, faces the proviso problem in some elementary cases. Section 3 develops incremental trivalent semantics in a way that addresses the proviso problem, with close attention to one intuition that underlies the Strong Kleene treatment: a presupposition of an operand is filtered if the value of a preceding operand independently determines the result of the propositional operation. Applying this condition globally, i.e. in all contextually salient situations, gives rise to a filtering condition that regulates the incremental semantic algorithm. This condition generalizes Karttunen's filtering conditions on specific connectives, thus avoiding the proviso problem. Section 4 addresses some central problems surrounding conditional presuppositions, context-sensitive filtering, and suspension. Conclusions are given in section 5.

\section{Setting the stage}

A presupposition of a sentence $S$ is pragmatically defined as a meaning component of $S$ that is taken for granted as common ground in all felicitous usages (von Fintel 2004). Thus, when a sentence $S_{1}$ has another sentence $S_{2}$ as one of its presuppositions, any participant who accepts $S_{1}$ as felicitous and true must accept $S_{2}$ as true as well. In this sense, presuppositions are like other entailments. For example, from sentence (2) below we infer the presupposition (3a) as much as we infer (3b).

(2) Sue's partner likes fishing.

(3) a. $\rightsquigarrow$ Sue has a partner.

b. $\Rightarrow$ Someone likes fishing.

Presuppositions are semantically distinguished from other entailments in the way they are inferred, or "projected", from complex sentences. ${ }^{1}$ Specifically, when a sentence is negated, its presuppositions may follow although its assertive content

1 Another distinction is also commonly suggested: falsifying (part of) a sentence's assertion makes the sentence false, whereas falsifying a presupposition of the sentence makes it exhibit a truth-value gap. von Fintel (2004) convincingly argues against using this common wisdom as an empirical criterion, mentioning that "the consensus view of presupposition experts" is that "people's intuitions about truth-value gaps [are] too unreliable to merit scientific attention" hence "argumentation cannot [...] proceed by simply polling naive speakers about whether sentences are false or neither true nor false". 
Presupposition and Trivalent Connectives

does not. For instance, from sentence (4) below we can infer (3a), but there is no way we can infer $(3 b){ }^{2}$

(4) Sue's partner doesn't like fishing.

From these standard considerations we derive the trivalent approach to presuppositions. In this approach, a sentence $S_{1}$ is said to presuppose a sentence $S_{2}$ if whenever $S_{1}$ is interpreted as true or false, $S_{2}$ is interpreted as true. Thus, $S_{2}$ is entailed by both $S_{1}$ and its negation. When the presupposition $S_{2}$ is not true, it is assumed that $S_{1}$ suffers a truth-value gap. Alternatively, falsity of $S_{2}$ may also be seen as resulting in $S_{1}$ having a third, "gappy" truth-value, which we here denote '*'. Thus, trivalent semantics respects the common intuition that any presupposition failure leads to a truth-value gap (note 1). Summarizing these ideas, we arrive at the following definitions (van Fraassen 1971: 154-5; Keenan 1973; Beaver 1997):

Trivalent interpretation: Sentences denote propositions that are interpreted as 1 (true), 0 (false) or * (failure) in any given model.

(6) Presupposition: We say that a proposition $\varphi$ presupposes a proposition $\psi$, and denote $\varphi \rightsquigarrow \psi$, if for every model $M$ : if $[[\varphi]]^{M} \neq *$ then $[[\psi]]^{M}=1$.

This notion of presupposition is a special case of standard Tarskian entailment (Nerlich 1965,Beaver 1997), defined by:

Tarskian entailment: We say that a proposition $\varphi$ entails a proposition $\psi$, and denote $\varphi \Rightarrow \psi$, if for every model $M$ : if $[[\varphi]]^{M}=1$ then $[[\psi]]^{M}=1$.

Entailments that are not qualified as presuppositions are referred to as assertions. Accordingly, we say that sentence (2) asserts (2b), or alternatively, that (2b) is part of (2)'s assertive content.

Presuppositions are semantically distinguished from assertions in that they may be inferred when triggered under negation (4). Similar "projections" of presuppositions are also manifest from antecedents of conditionals and under possibility modals, as in the following examples (Karttunen 1973):

a. If Sue brings her partner it will make Dan envious.

b. Possibly, Sue will bring her partner.

2 Projected presuppositions may be defeasible, unlike the simple presuppositions that they originate from (Abbott 2006). For example, while the presupposition (2) $\leadsto(3 a)$ is an indefeasible inference, the inference (4) $\rightsquigarrow(3 a)$ may be "suspended" or "canceled" under pragmatic pressures. We will get back to this point in section 4 , where it becomes theoretically crucial. 
From both (8a) and (8b) it is easy to infer that Sue has a partner. More generally, presuppositions are freely projected from environments that are non-veridical for other entailments. ${ }^{3}$

More complex projection patterns are observed with binary propositional constructions: conjunction, disjunction and conditionals. Let us first consider presupposition projection from conditional sentences, starting with the following example:

$$
\text { If Sue is in a good mood, she will bring her partner to the party. }
$$

Sentence (9) entails that Sue is in a relationship. Accordingly, we say that the presupposition of the consequent in (9) is projected out of the conditional. ${ }^{4}$ In this case there is no semantic relation between the antecedent of the conditional ("Sue is in a good mood") and the presupposition ("Sue has a partner") of the consequent. By contrast, let us consider the following example:

(10) If Sue is in a relationship, she will bring her partner to the party.

Unlike (9), the conditional in (10) does not presuppose that Sue has a partner. Thus, we say that the presupposition of the consequent in (10) is filtered. This filtering is assumed to be connected to the fact that the antecedent of (10) asserts the presupposition of the consequent: being in a relationship means having a partner. A similar case of filtering is observed in examples like the following:

If Sue is married, she will bring her partner to the party.

Similarly to (10), sentence (11) is consistent with situations in which Sue has no partner. Thus, the presupposition of the consequent is again filtered. As in (10), the antecedent of (11) entails the presupposition of the consequent, but in (11) the entailment is asymmetric, as having a partner does not entail being married.

To account for projection facts as in $(10,11)$, trivalent accounts often employ the Strong Kleene (SK) connectives in Table 1 (Beaver 1997; Beaver \& Krahmer 2001). When interpreting the value ' $*$ ' as "unknown", we can view these trivalent SK operators as derived from basic properties of the corresponding bivalent operators. Let us illustrate this using the material implication $\varphi \rightarrow \psi$. When $\varphi$ has the value 0 , the result of bivalent implication is 1 independently of the value of $\psi$. This property

$3 \mathrm{~A} n$-ary propositional operator op is called veridical in its $i$-th argument if it satisfies the entailment $o p\left(\varphi_{1}, \ldots, \varphi_{i}, \ldots, \varphi_{n}\right) \Rightarrow \varphi_{i}$ (Valencia, Van der Wouden \& Zwarts 1993; de Marneffe, Manning \& Potts 2012). Bivalent conjunction is veridical (in both arguments), whereas bivalent negation, implication and disjunction, as well as possibility modals are not veridical (in any argument).

4 When saying that a proposition $\psi$ is "the" presupposition of $\varphi$ we mean that $\psi$ is the maximal bivalent proposition that $\varphi$ presupposes. For instance, the maximal bivalent presupposition of the proposition for Sue's partner stopped smoking is the bivalent statement Sue has a partner, $x$, and $x$ used to smoke. Formally: a proposition $\psi$ is the maximal bivalent presupposition (in short: "the presupposition") of $\varphi$ if for every model $M:[[\varphi]]^{M} \neq *$ iff $[[\psi]]^{M}=1$, and $[[\varphi]]^{M}=*$ iff $[[\psi]]^{M}=0$. 
Presupposition and Trivalent Connectives

\begin{tabular}{|c|c|c|c|c|c|c|c|c|c|c|c|c|c|}
\hline$\neg$ & & $\wedge$ & 0 & 1 & $*$ & $V$ & 0 & 1 & $*$ & $\rightarrow$ & 0 & 1 & $*$ \\
\hline 0 & 1 & 0 & 0 & 0 & 0 & 0 & 0 & 1 & $*$ & 0 & 1 & 1 & 1 \\
\hline 1 & 0 & 1 & 0 & 1 & $*$ & 1 & 1 & 1 & 1 & 1 & 0 & 1 & $*$ \\
\hline$*$ & $*$ & * & 0 & $*$ & $*$ & * & $*$ & 1 & $*$ & $*$ & & 1 & $*$ \\
\hline
\end{tabular}

Table 1 Strong Kleene (SK) connectives: the rows/columns pertain to the value of the lefthand/righthand operand, respectively

is preserved in the definition of the trivalent operator. Thus, when $\varphi$ is 0 , the trivalent implication $\varphi \rightarrow \psi$ is defined as 1 even when the value of $\psi$ is ' $*$ '. Intuitively, this is justified because in the bivalent semantics of implication, when $\varphi$ is 0 it is possible to infer the result also when the value of $\psi$ is unknown, for the value $\varphi \rightarrow \psi$ is bound to be 1 anyway. By contrast, when $\varphi$ is 1 , we cannot determine the value of the bivalent implication $\varphi \rightarrow \psi$ without knowing the value of $\psi$. Accordingly, if $\varphi$ is 1 and $\psi$ is ' $*$ ', the result of SK implication is ' $*$ ' as well. A similar idea underlies the definitions of SK conjunction and disjunction.

In general, for all binary propositional operators $o p$, the SK truth-tables rely on the following definition:

(12) Value determination: A value $v$ of an operand determines the result of a binary operation op as $\mu$, if whenever $v$ is assigned to that operand, the result of the operation is $\mu$ independently of the value of the other operand.

When a value $v$ in the first operand determines the result of an operation as $\mu$, we say that $v$ left-determines the result as $\mu$. Similarly, when $v$ in the second operand determines the result as $\mu$, we say that $v$ right-determines the result as $\mu$. Thus, for the three bivalent propositional connectives we have:

- Conjunction is left-determined and right-determined by 0 as 0 .

- Disjunction is left-determined and right-determined by 1 as 1 .

- Implication is left-determined by 0 and right-determined by 1 , in both cases as 1 .

These value-determination properties are the key for defining the SK truth-tables.

Peters (1979) proposed an asymmetric version of the SK connectives, which is more closely related to the behavior of presuppositions with the propositional connectives in English. More recently, the reasoning that underlies the Strong Kleene/Peters tables has been used in incremental theories of projection (Schlenker 2008, 2009; Fox 2008; Mandelkern \& Romoli 2017, see also Mandelkern, Zehr, Romoli \& Schwarz 2019). One central idea of these theories is summarized by 
op: bivalent binary propositional operator

$S_{1}, S_{2}: \quad$ sentences with trivalent denotations $\left[\left[S_{1}\right]\right]$, [[S $\left.]\right]$

result: trivalent denotation $\left[\left[S_{1}\right.\right.$ op $\left.\left.S_{2}\right]\right]$

i. Evaluate the truth-value $\left[\left[S_{1}\right]\right]$.

ii. If $\left[\left[S_{1}\right]\right]=*$ then result $=*$, else:

iii. $\quad$ If $\left[\left[S_{1}\right]\right]$ left-determines $o p$ as $\mu$, then result $=\mu$, else:

a. Evaluate the truth-value $\left[\left[S_{2}\right]\right]$.

b. If $\left[\left[S_{2}\right]\right]=*$ then result $=*$, else:

c. $\quad$ result $=\left[\left[S_{1}\right]\right]$ op $\left[\left[S_{2}\right]\right]$.

\section{Algorithm 1: SK-based algorithm for presupposition projection}

Algorithm 1. For any two sentences $S_{1}$ and $S_{2}$ and a binary connective $o p$, Algorithm 1 evaluates the trivalent denotation of $S_{1}$ op $S_{2}$ using the trivalent (recursively given) denotations of $S_{1}$ and $S_{2}$ and the bivalent definition of $o p$.

To illustrate the way this incremental processing works, let us consider the following example, a variation of sentence (11):

(13) If the queen of Queensland is in a relationship, she will bring her partner to the party.

Intuitively, sentence (13) presupposes that there is a (unique) queen of Queensland, but not that she has a partner. Considering the way that Algorithm 1 behaves on the three possible truth-values of the antecedent, we observe the following cases:

A. The antecedent is true - there is a (unique) queen of Queensland and she is in a relationship. The truth of the antecedent does not allow us to determine the result of the bivalent implication, hence the consequent is evaluated in step (iii-a). The presuppositions of the consequent are satisfied: there is a Queenslandian queen, and she has a partner. Accordingly, a bivalent result is derived in step (iii-c), hence the conditional does not suffer a presupposition failure.

B. The antecedent is false - there is a (unique) queen of Queensland but she is not in a relationship. In this case the antecedent determines the bivalent implication as true. Thus, step (iii) defines the result of the conditional to be true without evaluating the consequent.

C. The antecedent fails - there is no (unique) queen of Queensland. In this case step (ii) defines the result to be a failure without evaluating the consequent. 
The only case where the truth-value of the consequent is considered is case A, where the presupposition that the queen has a partner is satisfied. This means that Algorithm 1 ignores presupposition failures of the consequent that ensue when the queen is not in a relationship. By contrast, the presupposition about the existence of a Queenslandian queen is projected, since case C makes failures of the antecedent lead to a failure of the conditional. This result is in line with the linguistic judgements.

When the processing of binary operations is assumed to go from left to right, Algorithm 1 gives Peters' asymmetric version of the SK tables. However, it is important to note that Algorithm 1 itself does not make any assumption about left-to-right processing. This becomes critical when considering antecedent-final conditionals like the following syntactic variation on (10):

(14) Sue will bring her partner to the party, if she is in a relationship.

Antecedent-final conditionals like (14) support filtering of the consequent's presupposition similar to the antecedent-initial conditional (10) (Mandelkern \& Romoli 2017 and references therein). Algorithm 1 does not specify which of the two sentences in a binary construction is the sentence $S_{1}$ that is processed first. This is something that a more general theory of semantic processing should specify. However, we may assume, as Mandelkern and Romoli do, that in both antecedent-initial conditionals (10) and antecedent-final conditionals (14), it is the antecedent that is processed first. This assumption accounts for the similar filtering behavior in (10) and (14). With respect to conjunctions and disjunctions, our analysis below will adopt usual assumptions about left-to-right semantic processing.

Algorithm 1 is an insightful account of presupposition filtering. It relies on the well-understood bivalent semantics of the propositional operators and on minimalist assumptions about semantic processing. At the same time, it suffers from a problem that has challenged theories of presupposition since the 1970s. To illustrate, let us reconsider sentence (9), restated below:

(15) If Sue is in a good mood, she will bring her partner to the party.

Hearing (15), we infer that Sue has a partner, but Algorithm 1 only expects a weaker inference. The algorithm only projects the presupposition of the consequent in (15) if the antecedent is true. However, if Sue is not in a good mood, the truth-value of the consequent sentence is not evaluated and the conditional is expected not to presuppose anything. This kind of problem for SK-based accounts of presupposition was recognized in Karttunen (1973). The same problem was also observed for later accounts (Karttunen 1974; Heim 1983) and reintroduced under the label of the proviso problem (Geurts 1996).

When it comes to conjunctions and disjunctions, Algorithm 1 shows similar advantages and disadvantages to those we have discussed in relation to conditionals. As for disjunctions, let us consider the following examples: 
(16) Either Sue is not in a relationship or (else) she will surely bring her partner to the party.

(17) Either Sue is unmarried, or (else) she will surely bring her partner to the party.

(18) Either Sue will stay at home tonight or (else) she will surely bring her partner to the party.

From sentence (16) we cannot infer anything about Sue's relationship: she may either have a partner or she may not. Sentence (17) is also indecisive on whether Sue has a partner: the sentence is consistent with situations where Sue is not in a relationship (hence unmarried) or where she has a partner (be it her spouse or not). It is natural to assume that in sentences (16) and (17), the presupposition that Sue has a partner is filtered because the assertion in the first conjunct supports an alternative scenario that can make the disjunction true without requiring that Sue is in a relationship (the first conjunct in (16) even requires that she is not). Algorithm 1 respects this intuition: in (16) and (17), the second conjunct is evaluated only when the first conjunct is false, i.e. in case Sue has a partner (16) (or furthermore a spouse, in the case of (17)). What Algorithm 1 does not expect is the complete absence of filtering in (18). If Sue stays at home, the algorithm does not expect sentence (18) to convey that she is in a relationship, which it univocally does. When it comes to disjunction, this is another instance of the proviso problem for Algorithm 1.

Moving on to conjunctions, let us consider the following example:

(19) Sue is in a relationship and will bring her partner to the party.

Intuitively, we may expect that since sentence (19) explicitly asserts that Sue is in a relationship, it cannot presuppose that. This expectation is supported by examples like the following:

(20) a. If Sue is in a relationship and brings her partner, it will surely make Dan envious.

b. Possibly, Sue is in a relationship and will bring her partner

In both (20a) and (20b), it is impossible to infer that Sue has a partner. This helps to classify the claim in (19) that Sue has a partner as part of the assertive content of this sentence rather than a presupposition. Accordingly, (19) is classified as yet another case of presupposition filtering. A similar case of filtering can be demonstrated in the following example, where the first conjunct asymmetrically entails that Sue has a partner:

(21) Sue is married and will bring her partner.

Algorithm 1 accounts for filtering in (19) and (21) similarly to what we saw with conditionals and disjunctions. Whenever the first conjunct in $(19) /(21)$ is false, the 
second conjunct is ignored; and when the first conjunct is true, the presupposition of the second conjunct is satisfied. Once more, Algorithm 1 is less successful when there is no semantic relation between the conjuncts:

(22) Sue will arrive and bring her partner.

Sentence (22) univocally presupposes that Sue has a partner. Algorithm 1 fails to predict that. Counter-intuitively, the algorithm only expects sentence (22) to presuppose that Sue has a partner if she arrives. This is again the proviso problem for Algorithm 1.

\section{The filtering condition}

The examples that were reviewed in section 2 involve conditionals, disjunctions and conjunctions. The regularities of the projection/filtering that are observed in these elementary cases are described in (Karttunen 1973: $(13,17,24))$ as follows:

(F1) In conditionals if $S_{1}$ then $S_{2}$ and conjunctions $S_{1}$ and $S_{2}$, a presupposition of $S_{2}$ is filtered if and only if $S_{1}$ entails that presupposition.

$\left(\mathrm{F}_{2}\right)$ In disjunctions $S_{1}$ or $S_{2}$, a presupposition of $S_{2}$ is filtered if and only if the negation of $S_{1}$ entails that presupposition.

These rules are illustrated above with the presupposition trigger Sue's partner. Similar regularities have also been demonstrated with other presupposition triggers: definite descriptions, factives (know/happy/regret that...), aspectual verbs (start, stop), additive adverbs (too, again) and clefts (it is NP who...).

When considered on the background of the Strong Kleene connectives, Karttunen's conditions point toward a generalization that does not invoke the proviso problem. To see that, let us first reconsider the relevant examples with conditionals:

a. If Sue is in a relationship (is married), she will bring her partner to the party. $(=10,11)$

$\not \rightarrow$ Sue has a partner - filtering of presupposition from second operand

b. If Sue is in a good mood, she will bring her partner to the party. $(=15)$

$\rightsquigarrow$ Sue has a partner - no filtering of presupposition from second operand

In sentence (23a) the antecedent entails the presupposition of the consequent. In (23b) there is no logical relation between the antecedent and that presupposition. Karttunen's condition $\left(F_{1}\right)$ relates this logical difference between (23a) and $(23 b)$ to the contrast in presupposition projection. This intuitive connection begs the question why filtering in conditionals should work this way. As we saw, previous methods that are inspired by the Strong Kleene semantics only give a partial answer 
to this question. Such accounts elegantly treat filtering in (23a) as a matter of the trivalent SK semantics of implication (Table 1) or incremental semantic processing (Algorithm 1). However, in (23b), previous SK-inspired methods fail to predict full projection of the antecedent's presupposition. This is an instance of the proviso problem that challenges SK-based treatments of conditionals. ${ }^{5}$

But is the proviso problem a fundamental challenge for the general principles of the SK system? One reason to doubt it arises once we observe that the proviso problem is tied with a localist view, which treats projection as a truth-functional phenomenon. Semantic accounts that lead to the proviso problem typically assume that in cases like (23), it is sufficient to evaluate the antecedent in a given situation in order to correctly handle presupposition projection from the consequent. By contrast, Karttunen's descriptive condition $\left(\mathrm{F}_{1}\right)$ relies on entailments between propositions, which can only be assessed globally by looking at a large class of relevant situations. Although conditions $\left(\mathrm{F}_{1}\right)$ and $\left(\mathrm{F}_{2}\right)$ may look ad hoc, we will now see that they can be reformulated as stemming from a globalist use of the SK-based notion of value determination. This leads to a more general filtering condition that does not suffer from the proviso problem.

To illustrate the idea, let us consider Karttunen's analysis of sentence (23a). In this case, the entailment from the antecedent to the presupposition of the consequent means that whenever the consequent fails the antecedent is false. Thus, here we have a consequent whose presupposition only fails in situations where the antecedent alone determines the result of the implication. According to the SK reasoning, the presupposition of the consequent in (23a) can be globally ignored, since the result of the implication can always be inferred when that presupposition fails. This accounts for the filtering in (23a). By contrast, in sentence (23b), the consequent may suffer a presupposition failure while the antecedent is true. This happens in cases where Sue is in a good mood but does not have a partner. In such cases, even Strong Kleene cannot "save" the result from failing. This means that the presupposition of the consequent in (23b) cannot always be ignored, as there are cases of presupposition failures in the consequent that can not be saved. In such cases, where there is no global way to filter a presupposition, Karttunen's description dictates that it must be globally projected.

To develop this reasoning further, we first formulate trivalent versions of Karttunen's conditions $\left(\mathrm{F}_{1}\right)$ and $\left(\mathrm{F}_{2}\right)$. Condition $\left(\mathrm{F}_{1}\right)$ sanctions filtering in conditionals and conjunctions where the sentential operand $S_{1}$ entails the maximal presupposition of $S_{2} \cdot{ }^{6}$ This means that in every model $M$ where $\left[\left[S_{1}\right]\right]^{M}$ is 1 , there is a bivalent

5 Methods relying on context changing operators (Stalnaker 1973; Karttunen 1974; Heim 1983) suffer from a similar problem.

6 For the sake of presentation here I assume that conditions $\left(F_{1}\right)$ and $\left(F_{2}\right)$ only regulate filtering of the maximal presupposition of $S_{1}$. In (Karttunen 1973) it is not completely clear whether these conditions 
truth-value to $S_{2}$, i.e. $\left[\left[S_{2}\right]\right]^{M} \neq *$. Equivalently, when $\left[\left[S_{2}\right]\right]^{M}=*$ we have $\left[\left[S_{1}\right]\right]^{M}=0$ or $\left[\left[S_{1}\right]\right]^{M}=*$. Thus, we reformulate $\left(\mathrm{F}_{1}\right)$ as follows:

(F ${ }_{1}^{\prime}$ ) In conditionals if $S_{1}$ then $S_{2}$ and conjunctions $S_{1}$ and $S_{2}$, the presupposition of $S_{2}$ is filtered if and only if in every model $M$ where $\left[\left[S_{2}\right]\right]^{M}=*$ we have $\left[\left[S_{1}\right]\right]^{M}=0$ or $\left[\left[S_{1}\right]\right]^{M}=*$.

Condition ( $\mathrm{F}_{1}$ ') sanctions filtering only if $S_{2}$ 's failure entails that $S_{1}$ is a falsity or a failure. Cases where $S_{1}$ is false allow us to determine the result of the implication/conjunction despite $S_{2}$ 's failure. Cases where both $S_{2}$ and $S_{1}$ fail may be considered "lost cases" for filtering: no non-arbitrary method can salvage the result from failing when both operands fail. Condition $\left(\mathrm{F}_{1}{ }^{\prime}\right)$ allows filtering of $S_{2}$ 's presupposition only if its failure is guaranteed to determine the result, ignoring such "lost cases".

Moving on to disjunctions, we can now state more directly how their projection/filtering behavior differs from that of implications and conjunctions. We restate Karttunen's condition $\left(\mathrm{F}_{2}\right)$ as follows:

(F2') In disjunctions $S_{1}$ or $S_{2}$, the presupposition of $S_{2}$ is filtered if and only if in every model $M$ where $\left[\left[S_{2}\right]\right]^{M}=*$ we have $\left[\left[S_{1}\right]\right]^{M}=1$ or $\left[\left[S_{1}\right]\right]^{M}=*$.

The underlined clause in $\left(\mathrm{F}_{2}{ }^{\prime}\right)$ highlights its difference from $\left(\mathrm{F}_{1}{ }^{\prime}\right)$. Keeping in mind that with disjunction it is truth of the first operand that determines the result rather than falsity, we are led to the following more general filtering condition: ${ }^{7}$

(24) Filtering Condition (first version): A proposition $\varphi$ op $\psi$ satisfies the filtering condition if every situation failing $\psi$ leads to a value of $\varphi$ that is either $*$ or determines the bivalent result of $\varphi$ op $\psi$.

The filtering condition in (24) is a trivalent generalization of Karttunen's (1973) specific observations. Let us now introduce this condition into Algorithm 1. In the revised Algorithm 2, we no longer "blindly" compute a bivalent result whenever the truth-value of the first sentence $S_{1}$ allows us to determine that result. Rather, Algorithm 2 only computes results in this way when the filtering condition holds (clause (iii)). Thus, in sentences $(23 \mathrm{a}, 19 / 21,16 / 17)$, where the filtering condition (24) holds, Algorithm 2 filters the presupposition in the second operand similarly to Algorithm 1. However, if the filtering condition does not hold, then any failure of the second sentence $S_{2}$ leads clauses (iii-a) and (iii-b) of Algorithm 2 to fail the result.

are also supposed to regulate filtering of non-maximal presuppositions (e.g. the presupposition Dan has a sibling of the sentence Dan's sister left). This point is important for the analysis of partial and selective filtering, discussed in section 4.

7 A generalization along these lines seems to be implied by the remarks in Karttunen (1973: 181-2). 
$o p: \quad$ bivalent binary propositional operator

$S_{1}, S_{2}: \quad$ sentences with trivalent denotations $\left[\left[S_{1}\right]\right]$, [[S $\left.]\right]$

result: trivalent denotation $\left[\left[S_{1}\right.\right.$ op $\left.\left.S_{2}\right]\right]$

i. Evaluate the truth-value $\left[\left[S_{1}\right]\right]$.

ii. If $\left[\left[S_{1}\right]\right]=*$ then result $=*$, else:

iii. If the filtering condition holds of $S_{1}$ and $S_{2}$, and $\left[\left[S_{1}\right]\right]$ left-determines op as $\mu$, then result $=\mu$, else:

a. Evaluate the truth-value $\left[\left[S_{2}\right]\right]$.

b. If $\left[\left[S_{2}\right]\right]=*$ then result $=*$, else:

c. $\quad$ result $=\left[\left[S_{1}\right]\right]$ op $\left[\left[S_{2}\right]\right]$.

Algorithm 2: SK-based algorithm for presupposition projection, including a filtering condition

For instance, as we saw, in sentence (23b) the filtering condition (24) does not hold. Accordingly, clause (iii) of Algorithm 2 evaluates $S_{2}$ independently of the value of $S_{1}$. This evaluation leads the algorithm to assign the value ' $*$ ' to the conditional sentence (23b) in all cases where Sue does not have a partner, independently of her mood. This strategy avoids the proviso problem that Algorithm 1 faces with sentences like (23b), (18) and (22).

So far, we have only considered the filtering condition in cases where the first operand does not include presupposition triggers. However, we should also consider cases of multiple triggers as in (13), which is restated below: ${ }^{8}$

(25) If the queen of Queensland is in a relationship, she will bring her partner to the party.

As we saw above, (25) only presupposes that Queensland has a (unique) queen, and is neutral with respect to whether she has a partner. Thus, the presupposition of the antecedent is projected, whereas another presupposition that is contributed by the consequent is filtered. As illustrated in the discussion of (13) above, Algorithm 1 accounts for this fact. This is also the case for Algorithm 2, as the filtering condition (24) holds for sentence (25). The antecedent of (25) Tarski-entails that there is a unique queen of Queensland and she has a partner. This is the presupposition of the consequent. Equivalently: any failure of the consequent either leads to a failure of the antecedent (when the queen of Queensland fails to refer) or to its falsity (when

8 Examples with multiple triggers that are more problematic for Algorithm 2 are discussed at the end of section 4 . 
Presupposition and Trivalent Connectives

the queen of Queensland successfully refers to a person who doesn't have a partner). Sentences $(26,27)$ below illustrate similar points on conjunction and disjunction:

(26) The queen of Queensland is in a relationship, and she will bring her partner to the party.

(27) Either the queen of Queensland is not in a relationship, or she will bring her partner to the party.

Like (25), sentences $(26,27)$ satisfy the filtering condition in (24). Accordingly, Algorithm 2 models the filtering and projection correctly in these cases as well.

\section{On conditional presuppositions}

Karttunen's (1973) filtering conditions and the generalized condition (24) treat projection and filtering as a matter of all-or-nothing: either a presupposition is projected out of a propositional construction or it is filtered. However, since Karttunen \& Peters (1979), it has been repeatedly argued that presupposition filtering is sometimes partial: part of the logical import of a presupposition may be filtered while the rest is projected. Consider for instance the following example by Mandelkern \& Rothschild (2018):

(28) If Buganda is a monarchy, then Buganda's king will be at the meeting.

The antecedent of sentence (28) does not logically entail the presupposition of the consequent: a monarchy may obviously have a queen and not a king. Therefore, condition (24) expects (28) to fully project the presupposition that Buganda has a king. This prediction is problematic: if (28) presupposed that, it would entail that Buganda is a monarchy. That would make the conditional in (28) quite odd, as it would make the antecedent trivially true, hence logically redundant. However, in normal contexts, (28) shows no trace of the rhetorical effect that we find in conditionals whose antecedent is implied to be true ("if I am not a complete idiot...").

To address this problem, many theories treat cases similar to (28) using the assumption that such sentences invoke conditional presuppositions. For instance, Algorithm 1 treats sentence (28) as presupposing (29) below: ${ }^{9}$

(29) If Buganda is a monarchy, then it has a king.

Intuitively, sentence (29) seems a more likely candidate for being a presupposition of (28) than the statement Buganda has a king that is projected by Algorithm 2. However, in order to evaluate better the significance of this point we need to

9 Conditional presuppositions are usually analyzed using material implication. Thus, (28) is assumed to presuppose that Buganda either is a non-monarchy or has a king. For simplicity, I here and henceforth paraphrase such implicative propositions using conditional sentences. 
also consider two other well-known problems. One is the effect of contextual inferences on filtering, the other is the problem of presupposition suspension, or "local accommodation".

\subsection{The effects of contextual inferences on filtering}

Discussing his filtering conditions, Karttunen (1973) pointed out that salient assumptions of speakers and hearers may boost filtering even in the absence of logical entailment. To illustrate this fact, let us consider the following contrast from (Beaver 2001: 246,278):

(30) a. If Jane takes a bath, Bill will be annoyed that there is no more hot water.

b. If Jane wants a bath, Bill will be annoyed that there is no more hot water.

In ordinary contexts, sentence (30a) does not presuppose that there is no more hot water. By contrast, sentence (30b) does presuppose that. Thus, filtering of the consequent's presupposition occurs in (30a) but not in (30b). Beaver notes that sentence (30a) is likely to be uttered if in the relevant household, having a bath may exhaust available hot water supply. In this likely context for (30a), speakers perceive a causal relation between situations where Jane takes a bath and situations where there is no more hot water. By contrast, in (30b) it is unlikely that speakers perceive any connection between Jane's wish to take a bath and the lack of hot water. This contrast is assumed to be the source of the easiness with which filtering occurs in (30a) vis à vis (30b). Following similar points in (Karttunen 1973: 182-5), Beaver proposes that filtering in conditionals does not only occur under logical entailment, but also when speakers and hearers perceive an inference between the antecedent and the presupposition of the consequent. In sentence (30a), perceiving a causal relation between the sentences Jane takes a bath and there is no more hot water appears to be sufficient for licensing filtering despite the absence of logical entailment. The fact that no parallel causal perception exists in (30b) is held responsible for the lack of filtering. This shows a limitation of the filtering condition in (24): although it correctly expects no filtering in (30b), it incorrectly makes the same prediction for (30a), ignoring the influence of the perceived causal relation. ${ }^{10}$

Karttunen (1973: 183) proposes to revise his filtering condition into a more fine-grained condition, which also takes "some (possibly null) set $X$ of assumed facts" into account. For instance, in ordinary contexts for sentences like (30a) and (30b), we may assume that taking a bath is likely to exhaust hot water supply,

10 Conditional presuppositions have the opposite merits and demerits. If we assume that the presupposition of $(30 \mathrm{a} / \mathrm{b})$ is the conditional if Jane takes/want a bath there is no more hot water, this might work for (30a) but would invoke the proviso problem for (30b): the latter sentence would incorrectly be expected to only presuppose that there is no more hot water in cases where Jane wants a bath. 
but merely wanting a bath is not likely to do that. At first sight this reliance on "assumed facts" may look stipulative. To be sure, it makes the mechanisms of presupposition projection harder to falsify. However, Karttunen's idea embodies a perfectly sensible view on entailment in natural language and how it is influenced by non-logical knowledge. Once we adopt the Tarskian view in (7), we necessarily rely on restricting the class of models that is being quantified over. Restrictions on models that usually get the most attention in formal semantics concern the interpretation of content words (quantifiers, conjunctions, modals, temporal expressions etc.) and grammatical constructions (negation, passives, questions etc.). Thus, when quantifying over models as in definitions (6) and (7) we silently assume that in some class of intended models, the interpretation of content words and "logical" grammatical constructions remains constant, in some logically proper sense of "constancy" (Sher 1991; Keenan 2001). To make this explicit, we must relativize the filtering condition (24) to a class of models. Stating filtering conditions in relation to a "set $X$ of assumed facts" amounts to the same view, but also lets the context restrict the class of intended models. This gives us the more formal version of (24) below:

(31) Filtering Condition (revised version): A proposition $\varphi$ op $\psi$ satisfies the filtering condition relative to a class $\mathscr{M}$ of models if for every model $M \in \mathscr{M}$ : if $[[\psi]]^{M}=*$ then either $[[\varphi]]^{M}=*$ or $[[\varphi]]^{M}$ determines the bivalent result of $\varphi$ ор $\psi$.

Stating the filtering condition in this way means that filtering can only be fully specified given a class of models $\mathscr{M}$. The logical relations in $\left(\mathrm{F}_{1}\right)$ and $\left(\mathrm{F}_{2}\right)$, which we used in our examples for filtering throughout section 3 , should be seen as a limiting case: these relations between propositions hold in any linguistically relevant class of models. However, the class of models $\mathscr{M}$ can also be used to encode contextually assumed facts, like the inference from the proposition "Jane takes a bath" to "there is no more hot water". Let us represent this weak contextual inference as follows:

\section{bath $-\rightarrow \neg$ hot_water}

The perceived causality in (32) may be easily invoked by merely uttering the two sentences "Jane takes a bath" and "there is no more hot water". This is observed in the causal interpretation of the conjunction Jane took a bath and there is no more hot water now. The processes that lead to this perceived causality have little to do with presupposition projection. Thus, in sentence (30a), we assume that the recognition of the implication (32) happens prior to applying any projection mechanism. In any class of models that respects the implication in (32), condition (31) expects filtering in (30a). Conversely, in any class where (32) is invalid, no filtering is expected to occur. Such invalidation of the implication (32) happens if the context explicitly negates it, as in the following example: 
(33) The hot water supply in Bill's place relies on gas heating, so that no single person could possibly take a bath that would exhaust the hot water. At present there's some problem with Bill's heating system. Not knowing that, Bill suggests Jane, who is staying at his place, to take a bath whenever she pleases. If Jane takes a bath, Bill will be annoyed (to hear from her) that there is no more hot water.

Here, where sentence (30a) is used in a context that explicitly denies (32), we easily infer from it that there is no more hot water. Thus, as soon as the assumption about the causal relation in (32) is dropped there is no filtering, and sentence (30a) shows a stronger presupposition than the conditional presupposition that Algorithm 1 uniformly derives for it. This is another instance of the proviso problem. By contrast, Algorithm 2 with the revised filtering condition (31) does not suffer from this problem, in the same way that it avoids the proviso problem in (30b).

\subsection{Presupposition suspension}

As we saw, presuppositions usually get projected from non-veridical environments. However, it is well-known that this kind of projection may be "cancelled" or "suspended" under certain pragmatic conditions, independently of the filtering mechanism discussed so far in this paper. Consider for instance the following example:

(34) There is no king of France. Therefore, the king of France is not hiding in this room. (von Fintel 2008)

In out-of-the-blue contexts, negative sentences like the king of France is not hiding in this room project the presupposition about a French king (cf. (4)). However, in the context of (34), which explicitly denies this presupposition, it does not appear. It is natural to hypothesize that the potential contradiction is the cause for the fact that (34) does not project the existence presupposition of the king of France (Gazdar 1979; Soames 1982). A common approach to such cases since Heim (1983) has been to assume that with many of the presupposition triggers (the "soft" triggers of Abusch 2002), presupposition projection leads to a systematic ambiguity. For instance, the sentence the king of France is not hiding in this room in (34) is assumed to be ambiguous between:

A. A default reading, obtained using the normal projection strategies. This reading presupposes that there is a (unique) king of France, and asserts that that person is not hiding in this room.

B. A secondary reading, obtained using a special procedure of presupposition suspension. This reading does not presuppose anything, and simply asserts that there is no king of France who is hiding in this room. 
When the secondary reading is attested, we describe it by saying that the presupposition of the default reading is suspended, or "locally accommodated".

In trivalent semantics it is easy to derive presupposition suspension is by applying Bochvar's assertion operator to the default reading (Beaver \& Krahmer 2001). This is the operator that maps 0 to 0,1 to 1 and $*$ to 0 , thus making any trivalent proposition bivalent, where ' $*$ ' is mapped to falsity. Applying Bochvar's operator in the scope of negation suppresses the presupposition in (34). This move is technically unproblematic. ${ }^{11}$ By contrast, a detailed linguistic analysis of the circumstances that license presupposition suspension is quite challenging. ${ }^{12}$ For our purposes here it is sufficient to note that pragmatic pressures as in (34) may cause suspension of presuppositions that otherwise get projected as a matter of course.

\subsection{Back to conditional presuppositions}

With this background, let us get back now to sentence (28) and to its purported conditional presupposition (29):

(35) a. If Buganda is a monarchy, then Buganda's king will be at the meeting. $(=(28))$

b. If Buganda is a monarchy, then Buganda has a king. (=(29))

Sentence (35b) is surely a likely conclusion from (35a). The critical theoretical question concerns the status of this conclusion. Should (35b) be analyzed as a presupposition of (35a) that is derived by the core projection mechanism, or are there other reasons to infer (35b) from (35a) $?^{13}$ According to Karttunen's line, this question can only be settled once we know which facts a speaker or hearer believes in. In terms of the filtering condition (31), we need to determine the class of intended models $\mathscr{M}$. Relative to classes of models where the conditional (35b) holds, condition (31) expects (35a) to show filtering, thus not to presuppose that

11 Formally, let $\varphi=$ there is a (unique) king of France and $\psi=$ there is a king of France who is hiding in this room. Let $\chi$ be the trivalent proposition that equals $*$ if $\varphi$ is false and equals $\neg \psi$ otherwise. The default reading of the king of France is not hiding is $\neg \chi$, whereas the secondary reading is $\neg B(\chi)$, where $B$ is Bochvar's operator. This secondary reading amounts to the bivalent proposition $(\neg \varphi) \vee(\neg \psi)$, which accounts for the reasoning in (34).

12 Previous work in Abusch (2002); Abbott (2006); Abrusán (2016), among others, considers many complex examples of suspension, e.g.:

(i) If you stopped smoking in 2001, you are eligible for a payment from Tobacco Indemnity Fund. (Abusch 2002, adapted from Kadmon 2001)

Sentence (i) suspends the presupposition that the addressee used to smoke prior to 2001. The reasons for this suspension are harder to analyze than in (34).

13 Mandelkern \& Rothschild (2018: 3) quote an unpublished note by Heim (2006), who asks whether a hearer of (35a) accommodates (35b) when (35a) is presented out-of-the-blue. 
Buganda has a king. However, if within some class $\mathscr{M}_{0}$ there are models where Buganda is a monarchy without a king, then no filtering is expected to occur in (35a) relative to $\mathscr{M}_{0}$. Such classes of models describe contexts where the conditional (35b) is not taken for granted. With such classes, Algorithm 2 derives for (35a) the presupposition that Buganda has a king. This should make (35a) pragmatically infelicitous, since the antecedent Buganda is a monarchy is satisfied. Accordingly, in a context where (35b) is not assumed, a hearer may consider (35a) to be incoherent. This incoherence may lead the hearer in one of two directions. First, even if the hearer does not know (35b) for a fact, the likelihood of this statement may lead her to contemplate the possibility that the speaker assumes (35b). According to the filtering condition (31), that would make the presupposition about Buganda's king disappear. In such a case, upon hearing (35a), the hearer may infer (or "accommodate") (35b) not because this sentence is technically presupposed by (35a), but because it is $a$ priori plausible, and accepting it saves (35a) from infelicity. Alternatively, the hearer may suspend the presupposition that Buganda has a king altogether. This would result in the following reading of (35a), without any presupposition:

(36) If Buganda is a monarchy, then Buganda has a (unique) king and Buganda's king will be at the meeting.

Either strategy avoids the undesirable projection of the proposition that Buganda has a king, while leading to the conclusion in (35b). The possibility of such interpretative strategies weakens the argument that (35b) must be hard-wired as a presupposition of (35a).

The discussion above rests on two inferences that example (35a) involves, independently of the question of conditional presuppositions:

(i) The entailment that any country with a king is a monarchy;

(ii) The weak implication suggesting that a stereotypical monarchy (in Africa?) may have a king as a matter of course.

As we saw, both factors leave the possibility open that we do not need to derive a conditional presupposition in order to account for the interpretation of (35a). Looking at more examples can help us to tease factors (i) and (ii) apart. To do that, let us first consider sentence (37) below (from Geurts 1996), and its variation in (38):

(37) If Theo is a scuba diver, then he will bring his wet suit.

(38) If Theo is blind, then he will bring his guide dog.

From sentence (38) it is hard to infer that Theo has a guide dog, but it is natural to infer from it that Theo has a guide dog if he is blind. Similarly to the weak 
implication (ii) in (35a), also in (38) it is easy to perceive a causal connection between the blindness of a person and his owning a guide dog. Unlike (35a), the implication that someone who has a guide dog should be blind is not an entailment like (i) but an inference based on world knowledge. However, this inference is so prominent in our world knowledge that the analysis of (38) can remain quite close to that of (35a). The situation in sentence (37) is somewhat trickier. According to Geurts (1996: 272), we can draw from sentence (37) the conclusion that Theo has a wet suit, but it is more likely to draw from it the weaker conclusion that Theo has a wet suit if he is a scuba diver. The logical situation is similar to Beaver's example (30a): there may be an a priori assumption that if Theo is scuba diver he has a wet suit. Unlike (35a) and (38), the implication does not go in the other direction, from wet suits to scuba divers (surfers and other water sportspersons also wear wet suits). Thus, no pragmatic deviance would emerge in (37) from the presupposition that Theo has a wet suit. This may be the reason for the possibility that this presupposition may optionally be projected.

A different situation is observed in the following example from (Heim 1983):

\#If John has children, then Mary will not like his twins.

There is no natural candidate for a presupposition in (39), as the sentence is infelicitous. To analyze (39), it is relevant that there is an entailment from having twins to having children, similar to the entailment (ii) in sentence (35a). However, (39) is different from (35a) in that there is no common sense implication (not even a weak one) that someone who has children should have twins. Heim (1983) attributes the oddity of (39) to the oddity of that implication, which according to her proposal should be a conditional presupposition of (39). However, the oddity of sentence (39) might also stem from the projection of the presupposition John has twins, which should render the sentence pragmatically deviant. I think that this analysis in terms of pragmatic deviance is advantageous to Heim's account, which relies on a contradiction between her postulated conditional presupposition and common world knowledge. However, pace Heim, even in out-of-the-blue contexts, there is nothing inherently wrong in presupposing unlikely conditionals. For instance (cf. Geurts 1996: 284):

Jane knows/has discovered that if John has children then he must have twins.

The presupposition that we infer (or "accommodate") from (40) is perhaps scientifically dubious, but it does not lead to the same infelicity as in (39). This gives reason to doubt Heim's claim that the infelicity of (39) should result from its purported conditional presupposition, rather than from a simple presupposition that entails the antecedent, as straightforward projection would expect.

The following example is a variation on Heim's example: 
(41) \#If John has a child, then Mary will not like his son.

This example is minimally different from (35a). In both cases we have a conditional whose consequent triggers a gender presupposition ("king"/"son") on top of the information in the antecedent ("monarch"/"child"). According to the present account, the difference in the felicity of (35a) and (41) is related to the a priori likelihood of the implication from monarch to king, as opposed to implausibility of the implication from child to son. For relevant discussion see further in (Lassiter 2012).

The last two logical possibilities that may be considered in this respect involve examples like the following:

(42) If Theo is smart, then he will bring his wet suit.

(43) If Theo has a wet suit, then Sue will know that he is a scuba diver.

Sentence (42) presupposes that Theo has a wet suit, as Algorithm 2 expects. This is another case where there is no a priori implication from the antecedent to the presupposition of the consequent. In addition, in (42) there is no inferential relation in the opposite direction either. In (43), the prominent presupposition is that Theo is a scuba diver. A weak assumption that scuba divers have wet suits is likely to be defeated in this case by that presupposition. Conversely, assuming that scuba divers have wet suits might defeat the presupposition by rendering the conditional pragmatically odd. This situation might lead to suspension, with the following secondary reading of (43):

(44) If Theo has a wet suit, then he is a scuba diver and Sue will know that he is a scuba diver.

Table 2 summarizes the implicational variety that we can observe in the examples above, some of whose have conditional inferences that have been classified as conditional presuppositions. We see that the critical factor for the emergence of such inferences is the assumption in (i): whether there is an a priori weak implication from the antecedent $\varphi$ to the presupposition $\psi^{\prime}$ of the consequent. When such an implication is plausible in out-of-the-blue contexts, the presupposition is filtered, as Karttunen proposed. Similarly to Karttunen's analysis, the current account does not see implications that are part of common knowledge as conditional presuppositions that are derived by the projection mechanism. Indeed, as we saw, in the cases where such a relevant weak implication is a priori unlikely, the presupposition is fully projected.

In the examples of Table 2, an implication or entailment (ii) from $\psi^{\prime}$ to $\varphi$ may be a factor that primes the weak implication (i). However, on its own an inferential relation as in (ii) does not immediately lead to suspension, at least not with presuppositions that are triggered by possessive descriptions. For instance, in Heim's example (39) we do not have a reading like (45): 
Presupposition and Trivalent Connectives

$\psi^{\prime}$ projected? assumptions

(i)

(ii)

\begin{tabular}{|c|c|c|c|c|}
\hline$(35 a)$ if $[\mathrm{B} \text { is a monarchy }]^{\varphi}$ & $\begin{array}{l}\text { then }[\mathrm{B} \text { 's king will arrive] } \\
\psi^{\prime}=B \text { has a king }\end{array}$ & no & $\varphi--\rightarrow \psi^{\prime}$ & $\psi^{\prime} \Rightarrow \varphi$ \\
\hline (38) if [T is blind $]^{\varphi}$ & $\begin{array}{l}\text { then [he'll bring his guide } \operatorname{dog}]^{\psi} \\
\psi^{\prime}=T \text { has a wet suit }\end{array}$ & no & $\varphi--\rightarrow \psi^{\prime}$ & $\psi^{\prime}--\rightarrow \varphi$ \\
\hline$\overline{(30 a) \text { if }[\mathrm{J} \text { takes bath }]^{\varphi}}$ & $\begin{array}{l}\text { then }\left[\mathrm{B} \text { annoyed no hot water }{ }^{\psi}\right. \\
\psi^{\prime}=\text { no hot water }\end{array}$ & no & $\varphi--\rightarrow \psi^{\prime}$ & $\psi^{\prime}-f \rightarrow \varphi$ \\
\hline$(37)$ if $[\mathrm{T} \text { is a scuba diver }]^{\varphi}$ & $\begin{array}{l}\text { then }\left[\text { he'll bring his wet suit] }{ }^{\psi}\right. \\
\psi^{\prime}=T \text { has a wet suit }\end{array}$ & & & \\
\hline (39) \#if [J has children $]^{\varphi}$ & $\begin{array}{l}\text { then }[\mathrm{M} \text { won't like his twins }]^{\psi} \\
\psi^{\prime}=J \text { has twins }\end{array}$ & - & $\varphi-f \rightarrow \psi^{\prime}$ & $\psi^{\prime} \Rightarrow \varphi$ \\
\hline (43) if [T brings wet suit $]^{\varphi}$ & $\begin{array}{l}\text { then [S knows } \mathrm{T} \text { is scuba diver] } \\
\psi^{\prime}=T \text { is scuba diver }\end{array}$ & yes & $\varphi-f \rightarrow \psi^{\prime}$ & $\psi^{\prime}--\rightarrow \varphi$ \\
\hline (42) if $[\mathrm{T} \text { is smart }]^{\varphi}$ & $\begin{array}{l}\text { then [he'll bring his wet suit] }{ }^{\psi} \\
\psi^{\prime}=\text { T has a wet suit }\end{array}$ & yes & $\varphi-f \rightarrow \psi^{\prime}$ & $\psi^{\prime}-f \rightarrow \varphi$ \\
\hline
\end{tabular}

Table 2 Sentences with/without entailments (' $\Rightarrow$ ') and weak implications $\left({ }^{\prime}--\rightarrow\right.$ ') from the antecedent to the presupposition of the consequent (i), and in the opposite direction (ii)

(45) If John has children, then he has twins and Mary will not like his twins.

This way of analyzing (39) would lead to an acceptable (though again, scientifically dubious) interpretation that the sentence does not easily show. Another way to illustrate that suspension does not play a role in such examples is to consider examples like the following (cf. Beaver 2001: 122-3):

(46) If Buganda is a monarchy and Buganda's king is at the meeting, then our president will be honored.

Sentence (46) easily leads to the conclusion that if Buganda is a monarchy it must have a king. This conditional inference is analyzed as a presupposition by Algorithm 1 and similar theories, in a reminiscent way to their analysis of (35a). When Algorithm 2 is used for analyzing sentences like (46), the conditional inference can be analyzed as resulting from a weak contextual implication, but not in terms of suspension. The reason is that suppression would lead to the following reading of (46), which does not support any conditional inference about the gender of Buganda's monarch:

(47) If Buganda is a monarchy and has a king, and Buganda's king is at the meeting, then our president will be honored. 
This does not mean that presupposition suppression never plays a role when analyzing conditional inferences from presuppositional sentences. Consider for instance the following examples:

(48) If John went out, then he regretted that he didn't take his coat.

(49) a. If John went out and regretted that the didn't take his coat, then he stopped somewhere to have a cup of tea.

b. Possibly, John went out and regretted that the didn't take his coat.

In (48) a prominent inference is that John did not take his coat if he went out. By contrast, in (49a-b) it is not clear that there is any inference like that. More likely, these sentences entertain the possibility that John went out, did not take his coat, and regretted it. This gives reason to believe that suppression plays a role in both (48) and (49), rather than conditional presuppositions.

To sum up, there is little doubt that sentences with presuppositions may lead to conditional inferences and to filtering patterns that are not directly expected by Karttunen's generalizations in $\left(\mathrm{F}_{1}\right)$ and $\left(\mathrm{F}_{2}\right)$. However, such effects do not give decisive support for hypotheses about conditional presuppositions. The claims that have been made so far about conditional presuppositions do not make it clear that we must bite the bullet of tackling the proviso problem that is invoked by proposals that generate such presuppositions. The clearest cases of conditional inferences, like Mandelkern and Rothschild's example (28), can also be explained using independently motivated principles or other relevant factors. Therefore, given the available evidence, Algorithm 2, which relies on the filtering condition in (31), seems like a safer bet than Algorithm 1, which does not rely on any independent filtering principle.

This is not to say that Algorithm 2 is without problems. So far, all the potential difficulties that have been discussed here regarding Algorithm 2 concern familiar claims about conditional presuppositions. The theoretical approaches that these claims come to support rely on a strategy that we may call partial projection: part of a presupposition can be projected while the rest is filtered. While the justification for this strategy is controversial, it is definitely correct that when it comes to sentences with multiple presupposition triggers, projection is not a matter of all or nothing. When two or more triggers are involved, a presupposition of one trigger may be projected while a presupposition of another trigger is filtered (Zuber 1975; van der Sandt 1988). For instance, let us consider the following sentences:

(50) If [Mary is in a relationship] ${ }^{\varphi}$, then [she will bring her partner to her brother's birthday $]^{\psi}$.

(51) Possibly, [Mary is in a relationship] ${ }^{\varphi}$ and [will bring her partner to her brother's birthday] ${ }^{\psi}$. 
Sentences (50) and (51) clearly presuppose that Mary has a brother. However, it is just as clear that they do not presuppose that Mary has a partner. We may call this phenomenon selective projection. This behavior is not predicted by condition (31): among the situations that make the operand $\psi$ in these sentences fail there are situations where Mary doesn't have a brother but has a partner. In such situations, the operand $\varphi$ is true, which does not determine the value of the implication/conjunction. Thus, condition (31) does not expect any filtering in (50) and (51), in disagreement with the facts. This kind of selective filtering is not directly described by Karttunen's original conditions $\left(\mathrm{F}_{1}\right)$ and $\left(\mathrm{F}_{2}\right) .{ }^{14}$ Sentences $(50)$ and $(51)$ illustrate that the projection of presuppositions from different triggers should be handled independently of each other. The question of how to incorporate this intuition into a proviso-free trivalent semantics is beyond the scope of this paper. ${ }^{15}$

\section{Conclusions}

Presuppositions are often thought of as a pragmatic matter, and there are many good reasons for that. However, the projection of presuppositions is connected to questions that are at the heart of formal semantic theory. We cannot separate inference with presuppositions from the meaning of the most prototypically "logical" elements of language - the propositional connectives. Against this familiar background, this paper tried to make a relatively modest point: the Strong Kleene intuition about the logical connectives is useful even if the proviso problem makes it impossible to embrace the Strong Kleene tables as the semantics of the natural connectives. The determinant values in the classic bivalent truth tables can predict presupposition projection even when the Strong Kleene tables are abandoned. Using determinant values, Karttunen's original conditions can be synthesized with the incremental view on the Strong Kleene semantics, so that the proviso problem does not emerge. The many examples that have been argued to support the derivation of conditional presuppositions do not give sufficient reason to eschew this synthesis.

14 The filtering conditions $\left(\mathrm{F}_{1}\right)$ and $\left(\mathrm{F}_{2}\right)$ do not specify which presuppositions of the second operand $S_{2}$ they apply to. For instance, the consequent $S_{2}$ of (28) presupposes that Buganda has a king, which entails Buganda is a monarchy. It seems that Karttunen (1973) would not like to classify Buganda is a monarchy as a presupposition of the consequent in (28). As van der Sandt (1988: 46-7) claims, such a view does not conform with a semantic definition like (6).

15 Algorithm 1 does not offer an immediate remedy. Indeed, in (50) this algorithm does not project the presupposition that Mary has a partner, but at the usual price of the proviso problem: Algorithm 1 incorrectly expects (50) to only presuppose that Mary has a brother if she has a partner. 


\section{References}

Abbott, Barbara. 2006. Where have some of the presuppositions gone? In Betty Birner \& Gregory Ward (eds.), Drawing the Boundaries of Meaning: NeoGricean Studies in Pragmatics and Semantics in Honor of Laurence R. Horn, 1-20. Philadelphia: John Benjamins.

Abrusán, Márta. 2016. Presupposition cancellation: explaining the soft-hard trigger distinction. Natural Language Semantics 24(2). 165-202.

Abusch, Dorit. 2002. Lexical alternatives as a source of pragmatic presuppositions. In Semantics and Linguistic Theory (SALT), vol. 12, 1-19.

Beaver, David I. 1997. Presupposition. In Johan van Benthem \& Alice ter Meulen (eds.), Handbook of Logic and Language, 939-1008. Amsterdam: Elsevier.

Beaver, David I. 2001. Presupposition and Assertion in Dynamic Semantics. Stanford, CA: CSLI Publications.

Beaver, David I. \& Emiel Krahmer. 2001. A partial account of presupposition projection. Journal of Logic, Language and Information 10(2). 147-182.

von Fintel, Kai. 2004. Would you believe it? The king of France is back! (presuppositions and truth-value intuitions). In Marga Reimer \& Anne Bezuidenhout (eds.), Descriptions and beyond, 315-341. Oxford: Oxford University Press.

von Fintel, Kai. 2008. What is presupposition accommodation, again? Philosophical perspectives 22(1). 137-170.

Fox, Danny. 2008. Two short notes on Schlenker's theory of presupposition projection. Theoretical Linguistics 34(3). 237-252.

van Fraassen, Bas C. 1971. Formal Semantics and Logic. New York: Macmillan.

Francez, Itamar. 2019. How not to project the satisfaction theory of projection (on Karttunen) or: Who has a proviso problem? In Cleo Condoravdi \& Tracy Holloway King (eds.), Tokens of Meaning: Papers in Honor of Lauri Karttunen, 187-198. Stanford: CSLI Publications.

Gazdar, Gerald. 1979. Pragmatics: Implicature, Presupposition, and Logical Form. New York: Academic Press.

Geurts, Bart. 1996. Local satisfaction guaranteed: A presupposition theory and its problems. Linguistics and Philosophy 19(3). 259-294.

Heim, Irene. 1983. On the projection problem for presuppositions. In Daniel P. Flickinger (ed.), West Coast Conference on Formal Linguistics (WCCFL), vol. 2, 114-125. Stanford: CSLI Publications.

Heim, Irene. 2006. On the proviso problem. Presentation to Milan Meeting, Gargnano.

Kadmon, Nirit. 2001. Formal Pragmatics: Semantics, Pragmatics, Presupposition, and Focus. Blackwell.

Karttunen, Lauri. 1973. Presuppositions of compound sentences. Linguistic Inquiry 
Presupposition and Trivalent Connectives

4(2). 169-193.

Karttunen, Lauri. 1974. Presupposition and linguistic context. Theoretical Linguistics 1(1-3). 181-194.

Karttunen, Lauri \& Stanley Peters. 1979. Conventional implicature. In Choon-Kyu Oh \& David A. Dinneen (eds.), Syntax and Semantics, vol. 11, 1-56. New York: Academic Press.

Keenan, Edward L. 1973. Presupposition in natural logic. The Monist 57(3). 344-370.

Keenan, Edward L. 2001. Logical objects. In C. Anthony Anderson \& Michael Zelëny (eds.), Logic, Meaning and Computation: Essays in Memory of Alonzo Church, 151-183. Amsterdam: Kluwer.

Lassiter, Daniel. 2012. Presuppositions, provisos, and probability. Semantics and Pragmatics 5. 1-37.

Mandelkern, Matthew. 2016. Dissatisfaction theory. In Semantics and Linguistic Theory (SALT), vol. 26, 391-416.

Mandelkern, Matthew \& Jacopo Romoli. 2017. Parsing and presuppositions in the calculation of local contexts. Semantics and Pragmatics 10. Early access.

Mandelkern, Matthew \& Daniel Rothschild. 2018. Independence day? Journal of Semantics 36(2). 193-210.

Mandelkern, Matthew, Jérémy Zehr, Jacopo Romoli \& Florian Schwarz. 2019. We've discovered that projection across conjunction is asymmetric (and it is!). Linguistics and Philosophy Published online.

de Marneffe, Marie-Catherine, Christopher D. Manning \& Christopher Potts. 2012. Did it happen? The pragmatic complexity of veridicality assessment. Computational Linguistics 38(2). 301-333.

Mayr, Clemens \& Jacopo Romoli. 2016. A puzzle for theories of redundancy: Exhaustification, incrementality, and the notion of local context. Semantics and Pragmatics 9. 1-48.

Nerlich, George. 1965. Presupposition and entailment. American Philosophical Quarterly 2(1). 33-42.

Peters, Stanley. 1979. A truth-conditional formulation of Karttunen's account of presupposition. Synthese 40(2). 301-316.

van Rooij, Robert. 2007. Strengthening conditional presuppositions. Journal of Semantics 24(3). 289-304.

van der Sandt, Rob A. 1988. Context and Presupposition. New York: Croom Helm.

Schlenker, Philippe. 2008. Be articulate: A pragmatic theory of presupposition projection. Theoretical Linguistics 34(3). 157-212.

Schlenker, Philippe. 2009. Local contexts. Semantics and pragmatics 2. 1-78.

Schlenker, Philippe. 2011. The proviso problem: a note. Natural Language Semantics 19(4). 395-422. 
Sher, Gila. 1991. The Bounds of Logic: a Generalized Viewpoint. Cambridge, Massachusetts: MIT Press.

Singh, Raj. 2007. Formal alternatives as a solution to the proviso problem. In Semantics and Linguistic Theory (SALT), vol. 17, 264-281.

Soames, Scott. 1982. How presuppositions are inherited: A solution to the projection problem. Linguistic Inquiry 13(3). 483-545.

Stalnaker, Robert. 1973. Presuppositions. Journal of Philosophical Logic 2(4). 447-457.

Valencia, Víctor Sánchez, Ton Van der Wouden \& Frans Zwarts. 1993. Polarity, veridicality, and temporal connectives. In Amsterdam Colloquium, vol. 9, .

Winter, Yoad. 2019. Presupposition projection and repair strategies in trivalent semantics. In Mathematics of Language (MOL), vol. 16, 27-39. Toronto, Canada: Association for Computational Linguistics. https://www.aclweb.org/anthology/ W19-5703.

Zuber, Ryszard. 1975. On the semantics of complex sentences. Tech. rep. Indiana University Linguistics Club Bloomington, Indiana.

Yoad Winter

Utrecht Institute of Linguistics OTS

Utrecht University

Trans 10

3512 JK Utrecht

The Netherlands

y.winter@uu.nl 